alone; children are bombarded everywhere - whether as part of the supposedly healthy free school meals or at friends' houses and parties. We live in a culture where we use junk food as bribery, reward and a pacifier for our young. Until the culture and the environment we live in changes, then I do not see the situation improving. To get environment and behaviour change, I see no other option than government regulation, much like we have for tobacco and alcohol. We cannot expect the food companies to change themselves.

As a dental profession, I understand the need to 'educate' the public, and these campaigns should be done. However, information alone - I find often interpreted as lecturing and condescending - rarely induces behaviour change. ${ }^{3}$ With this in mind, we should not lose momentum and loudly and publicly continue to lobby government to introduce regulation to curb processed junk food in general, especially when targeted to the most precious and impressionable in our society, the best asset we have, our children.

S. Nolan, by email

1. Al-Mazyad M, Flannigan N, Burnside G, Higham S, Boyland E. Food advertisements on UK television popular with children: a content analysis in relation to dental health. Br Dent J 2017; 222: 171-176.

2. O'Dowd A. 2015. Type 2 diabetes prevention programme is needed to tackle projected five million rise in cases. BMJ 2015; 351: h4648. doi: 10.1136/bmj.h4648.

3. Walls H L, Peeters A, Proietto J, McNeil J J. Public health campaigns and obesity - a critique. BMC Public Health 2011; 11: 136.

\section{DOI: 10.1038/sj.bdj.2017.292}

\section{Oral cancer}

\section{Indian pandemic}

Sir, the Indian National Cancer Registry Programme report shows worrying rises in cancers of the upper aero-digestive tract (mouth, tongue, oro-pharynx, hypopharynx, larynx and oesophagus) among both sexes as important sites for undertaking risk factor research and implementing early detection programmes. ${ }^{1}$

The Global Adult Tobacco Survey India, conducted in 2009-10, revealed that $35 \%$ of adults used tobacco. ${ }^{2}$ Tobacco-related cancers are expected to constitute $30 \%$ of the total cancer burden by $2020 .{ }^{1}$ It is important to elevate smokeless tobacco, areca nut and oral cancer as an even greater problem than smoking for the Indian nation, and South Asia. The Indian subcontinent accounts for one third of the global burden of cancers of lip and oral cavity.
Cancers of mouth and tongue, taken together, overshadow cancer of lung. ${ }^{1}$ Likewise, in other cities of India like Delhi, Mumbai, Aurangabad and Kollam, after lung cancer, cancer of mouth [excluding tongue] is the second most common cancer among males. The projected burden of cancers among males by the year 2020 in India shows the number of cases will be lung $(102,300)$, mouth $(99,495)$, prostate $(61,222)$, tongue $(60,669)$ and larynx $(36,079)$. Cumulatively, this makes 'oral cancer' the leading cancer site for men in most of India. ${ }^{1}$

Improved public health education and promotion is vital, as are top down policy approaches such as those of the Framework Convention on Tobacco Control, extended to include all forms of smokeless tobacco. Much excellent work on the control of the continuing pandemic of oral cancer in India is ongoing ${ }^{3}$ and we write to draw these issues to the attention of clinicians, public health specialists and policy makers.

\section{B. Gupta, N. W. Johnson, Queensland, Australia}

1. Indian Council for Medical Research. Three-Year Report of Population Based cancer Registries 2012-2014. Bengaluru, India: National Centre for Disease Informatics and Research-National Cancer Registry Programme, 2016. Available at: http://www.ncrpindia.org.

2. Ministry of Health and Family Welfare, Government of India. Global Adult Tobacco Survey India 2009-2010: Country Report. New Delhi: International Institute for Population Sciences, 2010.

3. Sankaranarayanan R, Ramadas K, Amarasinghe H, Subramanian S, Johnson N. Oral cancer: prevention, early detection, and treatment. In Jamison D T, Nugent R, Gelband H, Horton S, Jha P, Laxminarayan R (eds). Disease control priorities. 3rd ed (Vol 3). Washington (DC): The International Bank for Reconstruction and Development/The World Bank, 2015.

DOI: 10.1038/sj.bdj.2017.293

\section{Saliva for biopsy}

Sir, salivary biomarkers have been identified in different tumours distant to the oral cavity including brain, pancreatic, breast, ovarian, lung, gastric, prostate, and oesophageal cancer. ${ }^{1}$ Saliva therefore represents a potential source of tumour markers (proteins, metabolites, mRNA, micro-RNA and microbial) but the development of this as an effective diagnostic modality requires further research. Because carcinogenesis is a complex process, it is necessary to know the molecular changes in primary tumour initiation, promotion and progression with a double objective: to detect early disease and to improve clinical management. For this, saliva could be a potential biofluid showing the heterogenecity of the tumour at different stages of the disease compared to tumour tissue and plasma. Research efforts should be directed to assess the diagnostic capacity of the different salivary tumour biomarkers as well as its biological function on the pathogenesis and progression of the disease. This will require the participation of different researchers (medical, dental, biologists, bioinformaticians, statisticians, engineers etc) and it is a matter of urgency to train such researchers and convince institutions about this excellent opportunity to finance projects in this field. New perspectives must be directed towards finding specific salivary biomarkers in cancer, with the aim of improving the diagnosis, prognosis and monitoring disease.

\section{O. Rapado-González, R. López López,}

M. M. Suárez-Cunqueiro, Santiago de Compostela, Spain

1. Rapado-Gonzalez O, Majem B, Muinelo-Romay L, López-López R, Suarez-Cunqueiro M M. Cancer salivary biomarkers for tumours distant to the oral cavity. Int J Mol Sci 2016; 17: DOI:10.3390/ijms17091531.

DOI: 10.1038/sj.bdj.2017.294

\section{Patient support}

\section{High-risk behaviour}

Sir, the case of National Aids Trust vs NHS England $^{1}$ in late 2016 stemmed a revolutionary breakthrough in the management of HIV in the UK which all medical professionals should be aware of. The court ruling deemed that the NHS can fund pre-exposure prophylaxis (PrEP) for those at risk of contracting HIV.

A 2014 government report ${ }^{2}$ stated there are about 107,800 individuals living with HIV in the UK with an overall prevalence of 2.8 per 1,000 population aged $15-59$ years. $\mathrm{PrEP}$ is a method to reduce the rate of transmission of HIV. The brand name Truvada consists of two anti-retroviral agents, emtricitabine and tenofovir. The logic is to give the medication to HIV negative patients prior to high risk behaviours to reduce the chance of later obtaining HIV. It can either be taken regularly ie one tablet per day, or only taken when needed, just prior to or following intercourse. The PROUD study ${ }^{3}$ indicated that there was a relative risk reduction of obtaining HIV of $86 \%$ in high risk sexual intercourse.

Despite the positive court ruling, the NHS has not yet started rolling out the medication en masse, largely due to the cost of the medication. A pack of 30 days of treatment costs $£ 355.73$. ${ }^{4}$ Instead a three-year trial starting in December 2016 consisting of 10,000 
participants will be conducted to assess the efficacy of the medication.

Even though the medication is not currently being funded, there are numerous sources available to patients to self-fund. Several websites have been set up to provide patients with resources to get medications, but this can lead to an increased black market supply of these medications. In order to combat this problem nationwide, many genitourinary clinics are now offering advice and support on purchasing PrEP in a safe and affordable manner. As dentists and other health professionals have an intimate relationship with patients, patients may disclose facts about risky behaviour to their health provider. Therefore, it is of utmost importance that the general dentist should be able to provide guidance and have some knowledge on where to obtain support for patients involved in high-risk behaviour.

\section{J. S. Chandan, S. Collins, T. Thomas,} Birmingham

1. Monaghan K Q, Leventhal Z. The Secretary of State for Health - and - The Local Government Association Defendant Interested Party Interested Party Mr Justice Green. 2005.

2. Aghaizu A, Anderson J, Birrell P et al. HIV in the United Kingdom: 2014 Report About Public Health England. 2014. www.gov.uk/phe (accessed 18 January 2017).

3. McCormack S, Dunn D T, Desai M et al. Pre-exposure prophylaxis to prevent the acquisition of HIV-1 infection (PROUD): effectiveness results from the pilot phase of a pragmatic open-label randomised trial. Lancet 2016; 387: 53-60.

4. British National Formulary. Truvada: British National Formulary. https://www.evidence.nhs.uk/formulary/bnf/ current/5-infections/53-antiviral-drugs/531-hiv-infection/nucleoside-reverse-transcriptase-inhibitors/tenofovir-disoproxil/with-emtricitabine/truvada (accessed 18 January 2017)

DOI: $10.1038 /$ sj.bdj.2017.295

\section{Orthodontics}

\section{Falling foul of standards}

Sir, I wish to address T. Kilcoyne's letter ${ }^{1}$ in which he asked me to apologise for stating that Fastbraces' advertising has been found by anybody to be misleading whilst also he also claimed that Fastbraces' claims follow evidence-based medicine.

I did not reply at the time as I felt it inappropriate to comment in detail on Advertising Standards Association (ASA) findings that were yet to be made public.

As of 8 March 2017, the ASA have published on their website (www.asa.org.uk) their final ruling on two dental practices that copied claims from Fastbraces' promotional literature onto their own practice websites.

The ASA found that this Fastbraces' advertising (which made claims about faster treatment, less painful treatment and less root resorption in comparison to other fixed appliance treatment) was 'misleading'. The ruling stated that these claims should not appear again in UK advertising without 'adequate substantiation' as a 'sufficient body of evidence' does not exist to justify them. There were 'significant methodological flaws' in the evidence that was provided to justify the Fastbraces' claims.

I feel that it is important for $B D J$ readers to realise that repeating the 'alternative facts' within the promotional literature of the Fastbraces company could lead to them falling foul of ASA and GDC standards.

I hope this will put an end to the matter and we can all accept that certain orthodontic systems are not magical and that competent dentists performing high quality orthodontic treatment is something we can all support.

N. Stanford, by email

1. Kilcoyne T. Orthodontics: monopolistic behaviours. Br Dent J 2016; 220: 558

DOl: 10.1038/sj.bdj.2017.296

\section{Multidisciplinary management}

\section{Botox and Parkinson's}

Sir, I work in general practice and came across a 60-year-old female patient who presented as an emergency in pain. I diagnosed acute apical periodontitis associated with the 33. After assessing her treatment options, patient choice was extraction. The interesting point to note was that the patient had significant bilateral jaw tremor (dystonia) secondary to Parkinson's. This was significantly more pronounced than the classic hand tremor associated with Parkinson's. This can often make dental treatment difficult to perform in a general practice not set up for sedation.

The patient reported that lignocaine-based anaesthetics tend to exacerbate her jaw tremor and so we opted for prilocaine. She also reported that her neurologist had obtained funding enabling him to inject botox into the facial muscles. On further questioning, she was adamant that this was a procedure being provided by her neurologist and not the maxillofacial team. C. Pedemonte et al. in 2015 reported a reduction in the signs and symptoms associated with oromandibular dystonia (bruxism, muscle pain and involuntary muscle contraction) with the application of botulinum toxin A. ${ }^{1}$ This raises the question of the scope of the application of botox and who should be providing it, especially where there is an overlap between our maxillofacial colleagues and neurologists in complex cases such as this. Furthermore, it raises the question of the effects different local anaesthetics have in patients with Parkinson's with dystonia. It seems that some clarity and guidelines are needed.

S. Olaore, M. Laudat, by email

1. Pedemonte C, Perez Gutierrez H, Gonzalez E, Vargas I, Laszo D. Use of onabotulinumtoxin A in post-traumatic oromandibular dystonia. J Oral Maxillofac Surg 2015; 73: 152-157.

DOI: 10.1038/sj.bdj.2017.297

\section{Dental trauma}

\section{Rare metabolic disorder}

Sir, an unusual case of dental trauma presented within the paediatric dental department. A 2-year-old boy was referred by his general dental practitioner regarding management for the avulsion of four incisors (61, 81, 71 and 72) following minor bumps and falls. The patient's parents were concerned regarding the early loss of his teeth. His primary incisors were traumatised on three occasions including falling on his mother's knee, knocking his teeth on the edge of a bouncy castle and a fall at home on a rug. His trauma history was inconsistent with the dental injuries suffered - raising caution to a possible non-accidental injury.

The parents had reported that their child is somewhat unstable with delayed walking and his health visitor has made a referral to a physiotherapist. In light of his trauma history and medical history, hypophosphatasia was suspected. His general medical practitioner had been contacted, blood tests were requested and referral to a to a paediatric endocrinologist made for further investigations.

Since his initial referral, the patient has been formally diagnosed with hypophosphatasia, an inherited metabolic disorder that has variable clinical presentation. The condition is extremely rare and is often linked with premature loss of teeth. This demonstrates the value of taking a good dental and medical history to aid diagnosis, which can significantly improve a child's ability to thrive.

\section{R. Chawla, A. Patel, S. Dunkley, Eastman Dental Hospital DOI: 10.1038/sj.bdj.2017.298}

